University of Wollongong

Research Online

Faculty of Engineering and Information

Faculty of Engineering and Information

Sciences - Papers: Part A

Sciences

$1-1-2016$

Comparison of rheological behaviors with fumed silica-based shear thickening fluids

\author{
Alain David Moriana \\ University of Wollongong, am358@uowmail.edu.au \\ Tongfei Tian \\ Tohoku University, tongfei@uow.edu.au \\ Vitor Sencadas \\ University of Wollongong, victors@uow.edu.au \\ Weihua Li \\ University of Wollongong, weihuali@uow.edu.au
}

Follow this and additional works at: https://ro.uow.edu.au/eispapers

Part of the Engineering Commons, and the Science and Technology Studies Commons 


\title{
Comparison of rheological behaviors with fumed silica-based shear thickening fluids
}

\begin{abstract}
Shear thickening fluids (STFs) of differing compositions were fabricated and characterised in order to observe the effect of varying chemical and material properties on the resultant rheological behavior. Steady shear tests showed that for a given carrier fluid and particle size exists an optimum weight fraction which exhibits optimal shear thickening performance. Testing also showed that increasing particle size resulted in increased shear thickening performance and its onset whilst altering the carrier fluid chemistry has a significant effect on the thickening performance. An explanation is provided connecting the effect of varying particle size, carrier fluid chemistry and weight fraction to the resultant rheological behavior of the STFs. Two STFs were chosen for further testing due to their improved but contrasting rheological behaviors. Both STFs displayed a relationship between steady and dynamic shear conditions via the Modified Cox-Merz rule at high strain amplitudes ( $\gamma 0 \geq 500 \%)$. Understanding the effects of particle and liquid polymer chemistry on the shear thickening effect will assist in 'tailoring' STFs for certain potential or existing applications.
\end{abstract}

\section{Keywords}

silica, fumed, behaviors, rheological, fluids, comparison, thickening, shear

\author{
Disciplines \\ Engineering | Science and Technology Studies
}

\section{Publication Details}

Moriana, A. D., Tian, T., Sencadas, V. \& Li, W. (2016). Comparison of rheological behaviors with fumed silica-based shear thickening fluids. Korea-Australia Rheology Journal, 28 (3), 197-205. 


\title{
Comparison of rheological behaviors with fumed silica-based shear thickening fluids
}

\author{
Alain D Moriana ${ }^{\mathrm{a}}$, Tongfei Tian ${ }^{\mathrm{b}}$, Vitor Sencadas ${ }^{\mathrm{a}}$, Weihua $\mathrm{Li}^{\mathrm{a}}{ }^{*}$

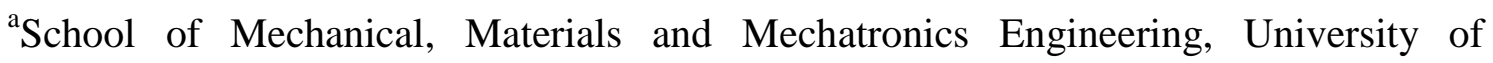 \\ Wollongong, Wollongong, NSW 2522, Australia \\ ${ }^{\mathrm{b}}$ Intelligent Fluid Control Systems Laboratory, Institute of Fluid Science, Tohoku \\ University, 2-1-1 Katahira, Aoba-ku, Sendai 980-8577, Japan
}

Email:weihuali@uow.edu.au

\begin{abstract}
Shear thickening fluids (STFs) of differing compositions were fabricated and characterised in order to observe the effect of varying chemical and material properties on the resultant rheological behavior. Steady shear tests showed that for a given carrier fluid and particle size exists an optimum weight fraction which exhibits optimal shear thickening performance. Testing also showed that increasing particle size resulted in increased shear thickening performance and its onset whilst altering the carrier fluid chemistry has a significant effect on the thickening performance. An explanation is provided connecting the effect of varying particle size, carrier fluid chemistry and weight fraction to the resultant rheological behavior of the STFs. Two STFs were chosen for further testing due to their improved but contrasting rheological behaviors. Both STFs displayed a relationship between steady and dynamic shear conditions via the Modified Coz-Merz rule at high strain amplitudes $\left(\gamma_{0} \geq 500 \%\right)$. Understanding the effects of particle and liquid polymer chemistry on the shear thickening effect will assist in 'tailoring' STFs for certain potential or existing applications.
\end{abstract}

Keywords: shear thickening fluids, rheological properties, viscosity, fumed silica, Cox-Mer 


\section{Introduction}

Shear thickening fluids (STFs) are a relatively new polymeric material that exhibits non-Newtonian behavior in which the viscosity of non-Newtonian fluids is dependent on the applied shear rate. STFs are unique as the viscosity of the solution increases with increasing shear rate, when a certain shear rate is applied, in a phenomenon known as "Shear Thickening". Specifically, above a critical shear rate, the viscosity of the STF exponentially increases with increasing shear force, often described as the "Shear thickening effect". Another unique aspect is the reversibility of this effect as the STFs will return to fluid state once the shear force is removed or reversed, making it advantageous as a reusable product in applications. Current applications with STFs include scenarios which require energy adsorption from sharp projectiles such as knife or spike attacks [1-11], acoustic and mechanical vibrational dampening control [12, 13] and in a passive capacity in other polymeric materials [14-16]. The theory of shear thickening includes an order-disorder transition of particles [17, 18] and formation of clusters, otherwise known as "hydroclusters" [19-22] that only form once the applied shear forces overcome particle repulsion forces.

Current research shows a gap in understanding the relationship between the rheological behavior of STF and their resultant effect in practical applications such as impact adsorption. It is crucial to understand the effect of systematically changing material properties on the STF's resultant rheology in order to manufacture a STF that suits the required application. A STF that thickens at very low shear stresses would be useful in sensitive applications such as acoustic dampening or low impact situations whilst a STF that thickens at higher shear stresses would be optimally used in high impact scenarios such as ballistic impacts. Barnes [23] gathered that the particles within a STF governed its rheological response and the shape, size, surface chemistry, volume fraction, 
dispersion in medium and reaction with the liquid all affected the STF's rheological properties. Ideally, by quantifying the effects of changing each variable on the resultant rheology, STFs can be fabricated to suit a certain application by simply altering its composition. In the present work, several STFs were produced from polyethylene glycol (PEG) and polypropylene glycol (PPG) with two different sized fumed silica particles at a range of weight fractions in the final STF. Rheological properties of the different samples were systematically characterised in order to determine the effect of varying certain material properties and their resultant rheological behavior. Theories as to how the varying material properties affect the formation of hydroclusters under steady shear were postulated.

\section{Experimental}

\section{Materials}

Three types of fumed silica were used in this experiment: AEROSIL® 130 (A130), AEROSIL $^{\circledR} 90$ (A90) and AEROSIL ${ }^{\circledR}$ OX 50 (OX50) which were all supplied by EVONIK Industries AG. All fumed silica particles were hydrophilic in nature, sensitive to moisture adsorption. The materials properties for all fumed silica variants can be viewed below in Table 1.

Table 1. Material properties of A130, A90 and OX50

\begin{tabular}{|c|c|c|c|}
\hline $\begin{array}{c}\text { Particle } \\
\text { name }\end{array}$ & $\begin{array}{c}\text { Primary particle } \\
\text { size }(\mathbf{n m})\end{array}$ & $\begin{array}{c}\text { BET-surface } \\
\text { area }\left(\mathbf{m}^{\mathbf{2}} / \mathbf{g}\right)\end{array}$ & $\begin{array}{c}\text { Tapped } \\
\text { density }(\mathbf{g} / \mathbf{l})\end{array}$ \\
\hline A130 & 16 & $130 \pm 25$ & 50 \\
\hline A90 & 20 & $90 \pm 15$ & 80 \\
\hline OX50 & 40 & $50 \pm 15$ & 130 \\
\hline
\end{tabular}

Before preparation, the fumed silica was place in a vacuum chamber for 24 hours and heated to $110^{\circ} \mathrm{C}$ to eliminate any moisture. 
PEG was used as carrier fluid with molecular weights of $400 \mathrm{~g} / \mathrm{mol}$. PPG was supplied in two differing molecular weights, 400 and $725 \mathrm{~g} / \mathrm{mol}$. All carrier fluids were supplied by Sigma-Aldrich. The main properties of PEG and PPG are presented in Table 2.

Table 2. Material properties of PEG400, PPG400 and PPG725

\begin{tabular}{|c|c|c|c|}
\hline $\begin{array}{c}\text { Polymer } \\
\text { name }\end{array}$ & $\begin{array}{c}\text { Molecular weight } \\
(\mathbf{g} / \mathbf{m o l})\end{array}$ & $\begin{array}{c}\text { Degree of } \\
\text { polymerisation }\end{array}$ & $\begin{array}{c}\text { Density at } \\
\mathbf{2 5}^{\circ} \mathbf{C}(\mathbf{g} / \mathbf{m L})\end{array}$ \\
\hline PEG400 & 400 & 9.09 & 1.13 \\
\hline PPG400 & 400 & 6.90 & 1.01 \\
\hline PPG725 & 725 & 12.50 & 1.01 \\
\hline
\end{tabular}

\section{STF fabrication processing}

For each STF, certain amounts of fumed silica were added to the various carrier fluid with a certain weight fraction (from $20 \mathrm{wt} \%$ to $55 \mathrm{wt} \%$ ). The silica was mixed with carrier fluid mechanically until it was apparent all fumed silica particles were satisfactorily blended into the carrier fluid in order to ensure an enhanced mixture of particles within the fluid. Once mixed, the mixture was placed in a vacuum chamber for one hour to remove any trapped air bubbles, then an additional mechanical stirring step was requested to ensure adequate distribution of silica within the STF.

\section{Materials characterisation}

Testing was completed with a parallel-plate Rheometer (MCR 301, Anton Paar, Germany) under steady shear and dynamic conditions with increasing angular frequency. A $20 \mathrm{~mm}$ diameter testing geometry (PP-20) was used with a gap of $0.8-$ 
$1.0 \mathrm{~mm}$ to ensure adequate filling of the STF over the testing disk. Viscosity was measured as a function of shear rate within the range of $0.1-1000 \mathrm{~s}^{-1}$ at $20^{\circ} \mathrm{C}$.

\section{Results and discussion}

Characterisation of STF mixtures

Fumed silica was chosen for testing due to its ability to induce shear thickening at relatively lower weight fractions compared with spherical silica and its increased particle-particle repulsion forces which lead to increased dispersion within the carrier fluid [24]. Transmission Electron Microscopic (TEM) images were taken for both fumed silica batches and are shown in Figure 1.

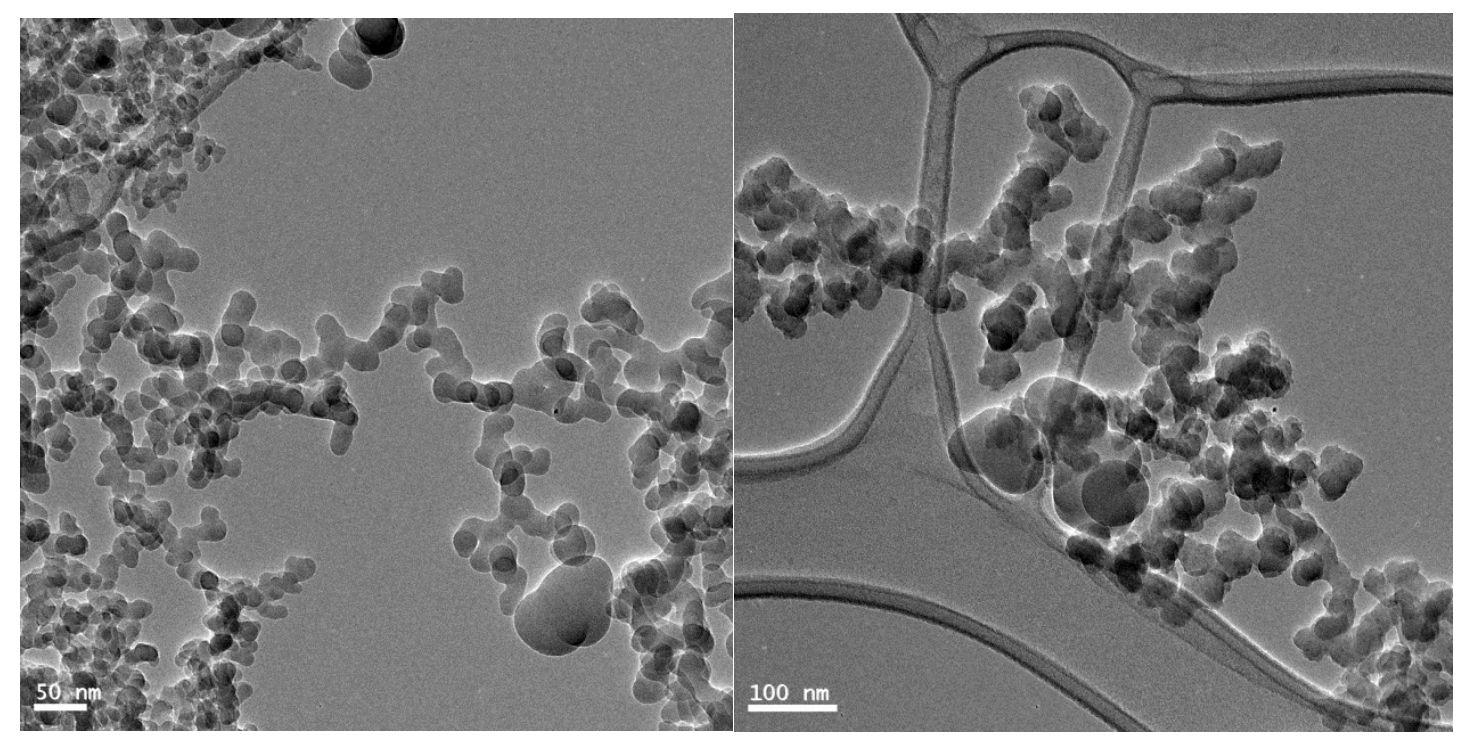

Figure 1. Representative TEM images of AEROSIL® 90 (Left) and AEROSIL® OX 50 (Right)

Preliminary testing was completed on various wt\% of both fumed silica batches in PEG400 and PPG400 to determine the optimum weight fraction of each batch that exhibit the highest shear thickening behavior. Fumed silica particles A90 was blended with PEG400, PPG400 and PPG725 in weight fractions between 20-40 wt\% and the resultant steady shear test results are shown below in Fig. 2 . 


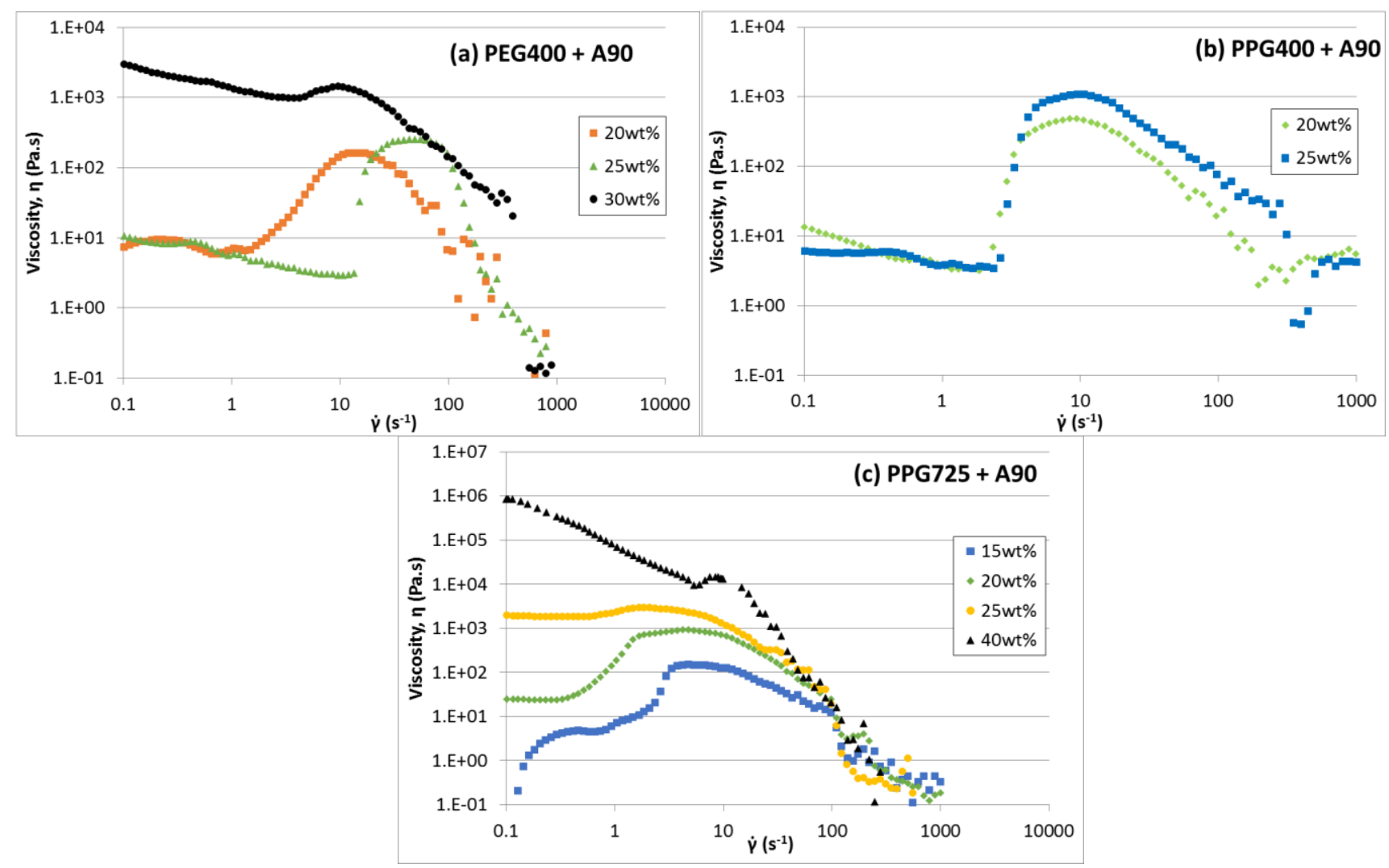

Figure 2. Viscosity measured as a function of shear rate under steady shear conditions for STF containing PEG400 + A90 (a), PPG400 + A90 (b) and PPG725 + A90 (c)

At low shear rates $\left(\dot{\gamma}<1 s^{-1}\right)$ all mixtures show shear thinning behavior which is characterised by decreasing viscosity with increasing shear rate. The onset of shear thickening at the critical shear rate, $\dot{\gamma}_{c}$, is an important point as it signifies the shear rate at which STF begins to thicken. Another important indication of the STF's ability to thicken is the ratio of the maximum viscosity to the viscosity at $\dot{\gamma}_{c}$, henceforth named 'Shear thickening ratio' (STR). With increasing wt\% in PEG400 + A90, different rheological behaviors are observed as $\dot{\gamma}_{c}$ and STR increases when comparing $20 \%$ and $25 \%$ STFs. At $30 \%$, there is a small increase in viscosity and overall shear thinning effect was observed, which is indicative of particle clustering, similar to that of a three 
dimensional network of an elastomer or gel. This was considered to be an oversaturated STF (figure 2a).

In PPG400 + A90 (Figure 2b), increasing the weight fraction shows significant increase in STR with infinitesimal change in $\dot{\gamma}_{c}$ and relatively larger in magnitude when compared to PEG400 + A90. We infer that the carrier fluid has a significant influence on the rheological properties of the STF when using the same fumed silica with same fractions. This is also evident when comparing 25wt\% A90 fumed silica in three different carrier fluids shown in Figure 3.

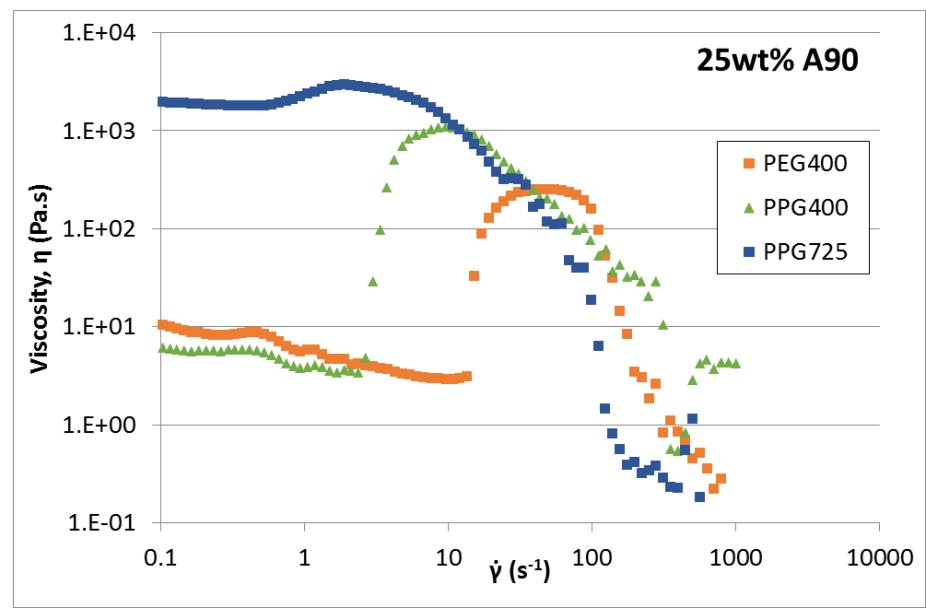

Figure 3. Viscosity measured as a function of shear rate under steady shear conditions for STF containing 25wt\% A90 using different carrier fluids

Increasing the molecular weight of PPG shows a predominately shear thinning mixture. A possible explanation is that the larger polymer chain of the PPG725 can result in entanglement with other PPG polymer chains within the solution, resulting in overall increased viscosity and difficulty to rearrange themselves under low to medium shear rates. As a consequence, STFs containing PPG725 were not further explored due their poor behavior as a possible application. 
STF mixtures containing A130 were then mixed into PEG400 and PPG400 at varying weight fractions. The results are shown below in Figure 4.

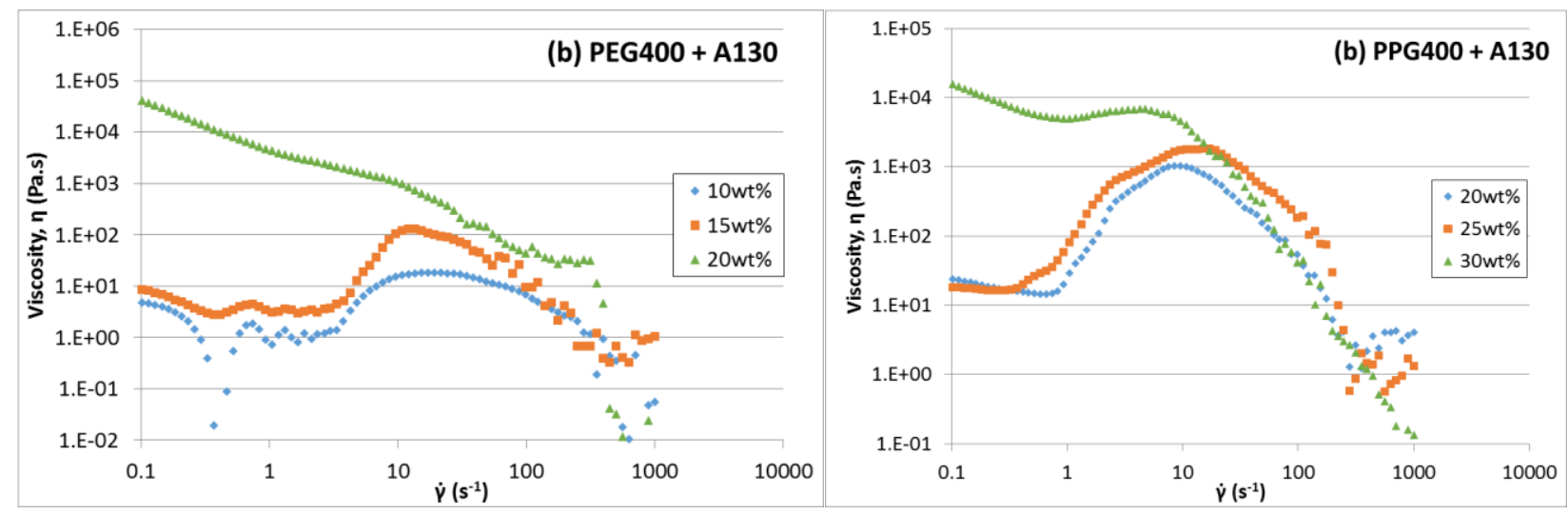

Figure 4. Viscosity measured as a function of shear rate under steady shear conditions for STF containing PEG400 + A130 (a) and PPG400 + A130 (b)

The rheological behaviours are significantly different when compared to using A90 fumed silica. For instance, the shear thickening phenomenon was observed in PEG400 + A130 at lower weight fractions and gel-like behaviour occurred at 20wt $\%$. Although the shear thickening effect was relatively poorer in PEG400 + A130 mixtures, fewer particles were required to observe this effect. In mixtures containing PPG400 + A130, the shear thickening effect was considerably unstable and the increase in viscosity past $\dot{\gamma}_{c}$ increased gradually and not sharply as observed in other STFs. The decreased particle size of A130 has a significant effect on the thickening effect of STFs. Wang and Wunder (2000) both quantified the differences of two types of fumed silica sizes and observed that "state of aggregation/agglomeration therefore increases with decreasing particle size" [25]. Gun'ko et al also stated "aggregation of primary particles...decrease...with decreasing specific surface area" [26]. Increased degrees of aggregation in smaller particles are proportional to increased aspect ratios and increased chances of interparticle clustering or 'hooking' to form a three-dimensional or 'gel' 
network. Gel-like behaviour typically exhibit a constantly shear thinning rheological response.

STF mixtures containing OX50 were then mixed into PEG400 and PPG400 at varying weight fractions and their resultant rheological behaviors are shown in Figure 5.

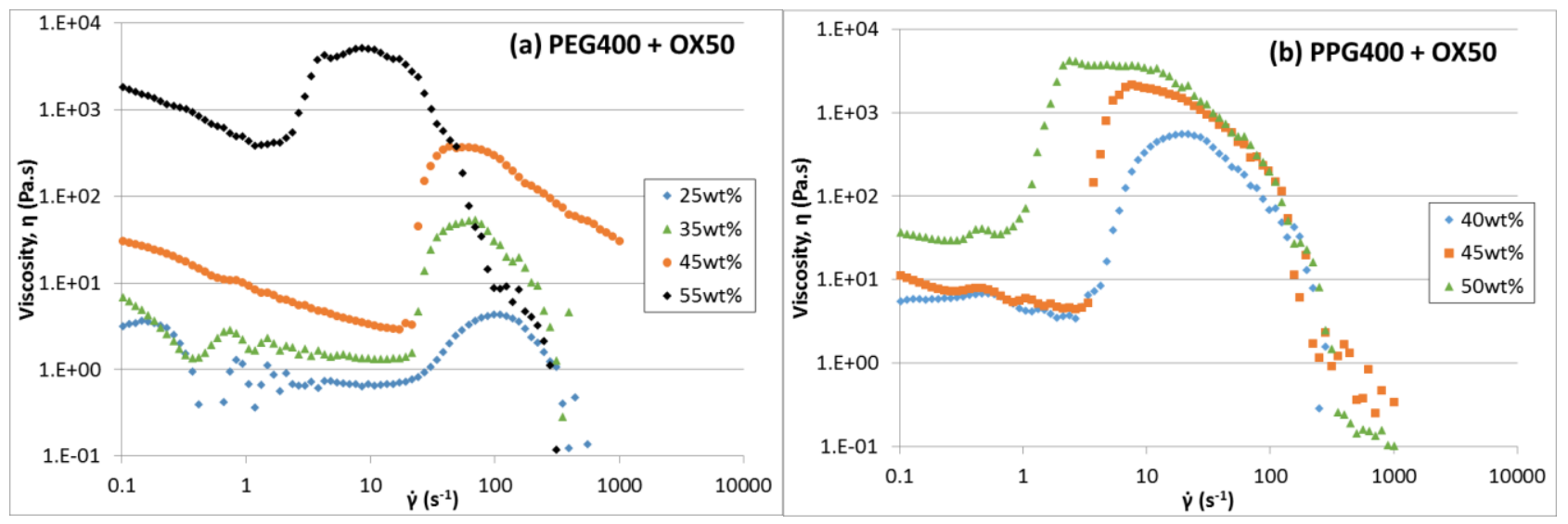

Figure 5. Viscosity measured as a function of shear rate under steady shear conditions for STF containing

$$
\text { PEG400 + OX50 (a) and PPG400 + OX50 (b) }
$$

Similar trends found in the STFs containing A90 can also be seen in STFs containing OX50. In PEG400 + OX50 and PPG400 + OX50, the optimum weight fraction was found to be $45 \mathrm{wt} \%$ which shows a good combination of $\dot{\gamma}_{c}$ and STR, increasing further resulted deterioration in performance, trending more to gel-like behavior (Figure 5).

From these preliminary results; two STF mixtures were chosen based on their superior rheological behaviors: PEG400 $+45 \mathrm{wt} \%$ OX50 and PPG400 $+45 \mathrm{wt} \%$ OX50 henceforth known as $\mathrm{STF}_{\mathrm{PEG}}$ and $\mathrm{STF}_{\mathrm{PPG}}$ respectively (Figure 6). 


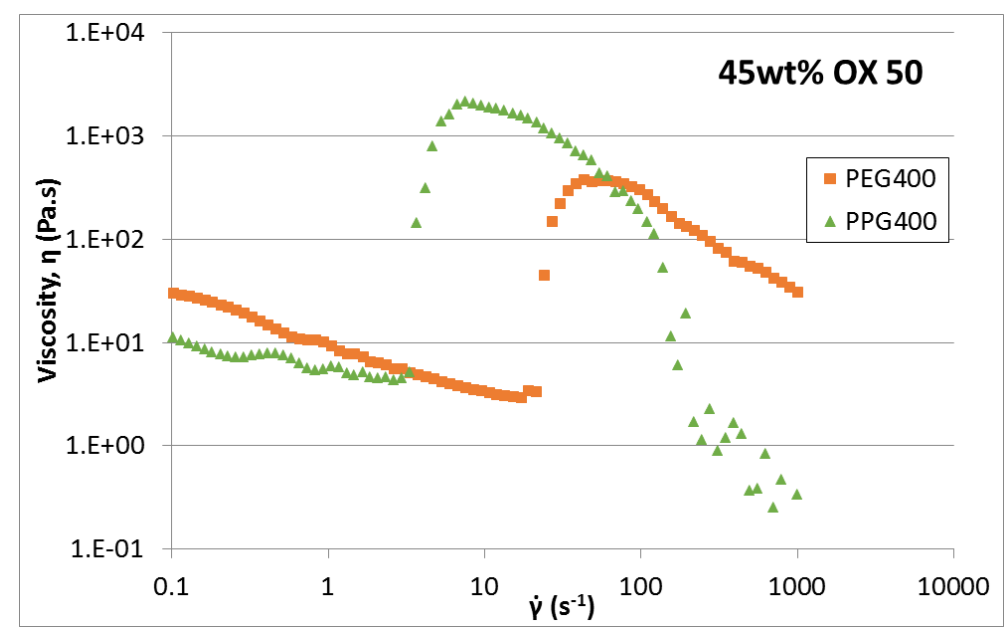

Figure 6. Viscosity measured as a function of shear rate under steady shear conditions for STF containing 45wt\% OX50 using different carrier fluids

\section{Discussion on variables and rheology of STF}

Barnes [23] stated that "All suspensions of solid particles will show the phenomenon [Shear thickening effect]". From this, we gather that the variables relating to particles including size, weight/volume fraction, surface chemistry and shape all have an effect on the resultant rheology when mixed with a fluid. To some extent, the reaction between the carrier fluid and the particles is also worth considering when attempting explaining the effect the relationship between physical variables and rheology. From this, a relationship can be developed that can quantify the effect of differing particle and carrier fluid material properties on the resultant rheological behavior of the STFs.

\section{Weight fraction}

During fabrication, the polymer chains within the carrier fluid react with the surface hydroxyl groups on the fumed silica (silanol groups) via hydrogen bonding. The resultant reactions between polymer and silica surface results in the formation of a layer of polymer surrounding fumed silica called a "solvation layer" [24, 26-28]. This was 
initially proposed by Raghavan et al. (2000) in which they stated "in strongly hydrogenbonding liquids, a solvation layer is envisioned to form on the silica surface through hydrogen bonding between liquid molecules and surface silanol groups." [27]. The solvation layer was also used to explain the results found by $\mathrm{He}$ et al (2015) when combining porous nanosilica particles in ethylene glycol [29]. This hypothetical solvation layer around silica would increase interparticle distancing resulting in increased deflocculation and interparticle repulsion forces. By increasing weight fraction of fumed silica, there is a larger readily reactive silanol surface area. As shown in Figures 3, 4, 5 and 6, there exists an optimum weight fraction of fumed silica, regardless of size, in which the shear thickening effect is pronounced. We suggest the remaining carrier fluid that has not reacted with the silanol surface groups act as lubrication between the fumed silica, allowing for increased particle distance and mobility to rearrange whilst under the applied shear force. Thus the conclusion can be made that a STF with a particle weight fraction below that of its optimum value will result in increased $\dot{\gamma}_{c}$ and decreased STR. This could be due to the larger interparticle distancing due to the abundance of unreacted liquid polymer chains present, requiring larger external forces to overcome this separation distance and the interparticle repulsion forces simultaneously. Inversely, by increasing the weight fraction beyond the optimum weight fraction, the STF is deprived of lubrication from unreacted polymer chains which results in entanglement of particle clusters leading to complete disappearance of the shear thickening effect. This optimum weight fraction was more dependent on the size of the fumed silica and less on the chemistry of the carrier fluid. A graphical example of this theory can be seen in Figure 7 where the critical shear rate and shear thickening ratio $\left(\eta_{\max } / \eta_{c}\right)$ were plotted against weight fraction. It is apparent that the critical shear rate of STFs containing PEG400 at varying weight 
fractions was significantly larger than STFs containing PPG400 and PPG725. The effect of differing carrier fluid chemistry is further examined below.

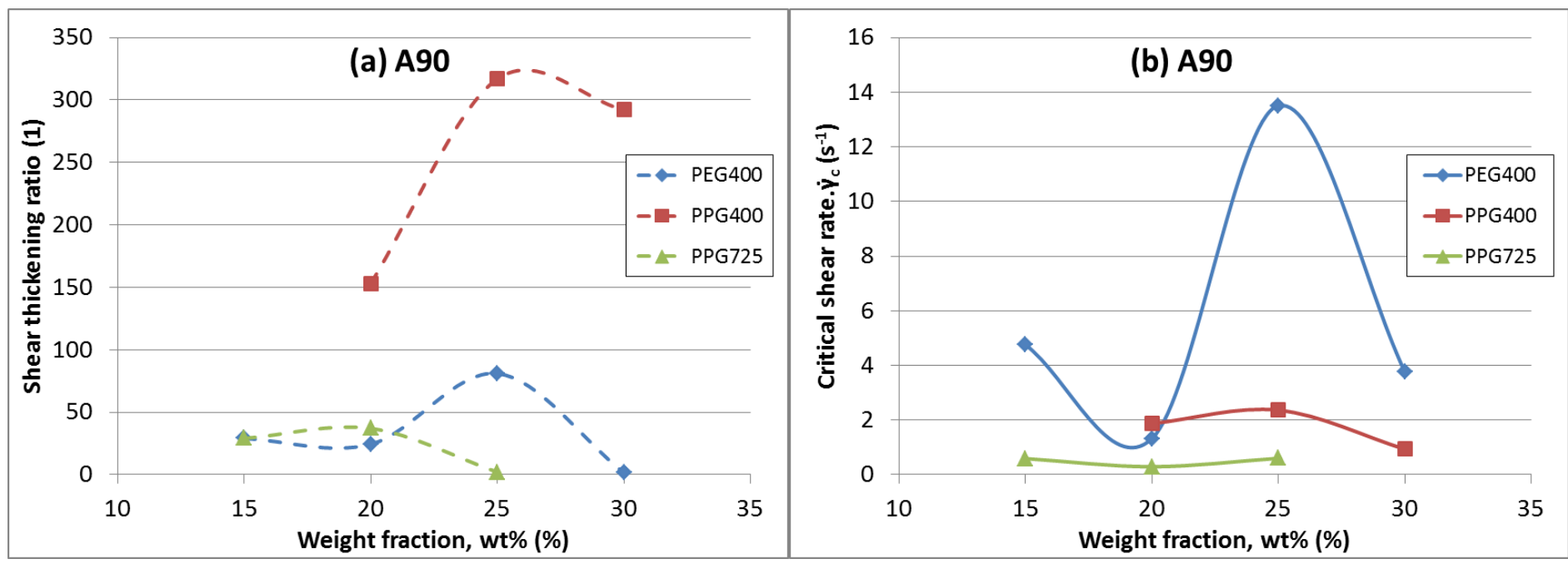

Figure 7. Shear thickening ratio (a) and critical shear rate (b) measured as a function of weight fraction mixed with A90 fumed silica under steady shear conditions

\section{Particle size}

Comparing the material properties of A90 and OX50, the size of fumed silica is inversely proportional to its BET-surface area, which is indicative of the exposed silica surface area available to bond with the carrier fluid polymer chains. Also, the optimum weight fraction for STFs containing smaller fumed silica was found to be relatively lower than that for STFs containing larger fumed silica. This implies that an 'Effective surface area' exists in which particle separation and lubrication is at their most effective state. This is further supported as the optimum weight fraction of OX50 is almost twice that of A90, and almost three times that of A130 which also coincides with the surface area of OX50 almost halving that of A90 and a third of A130. A graphical demonstration can be seen in Figure 8 below. 


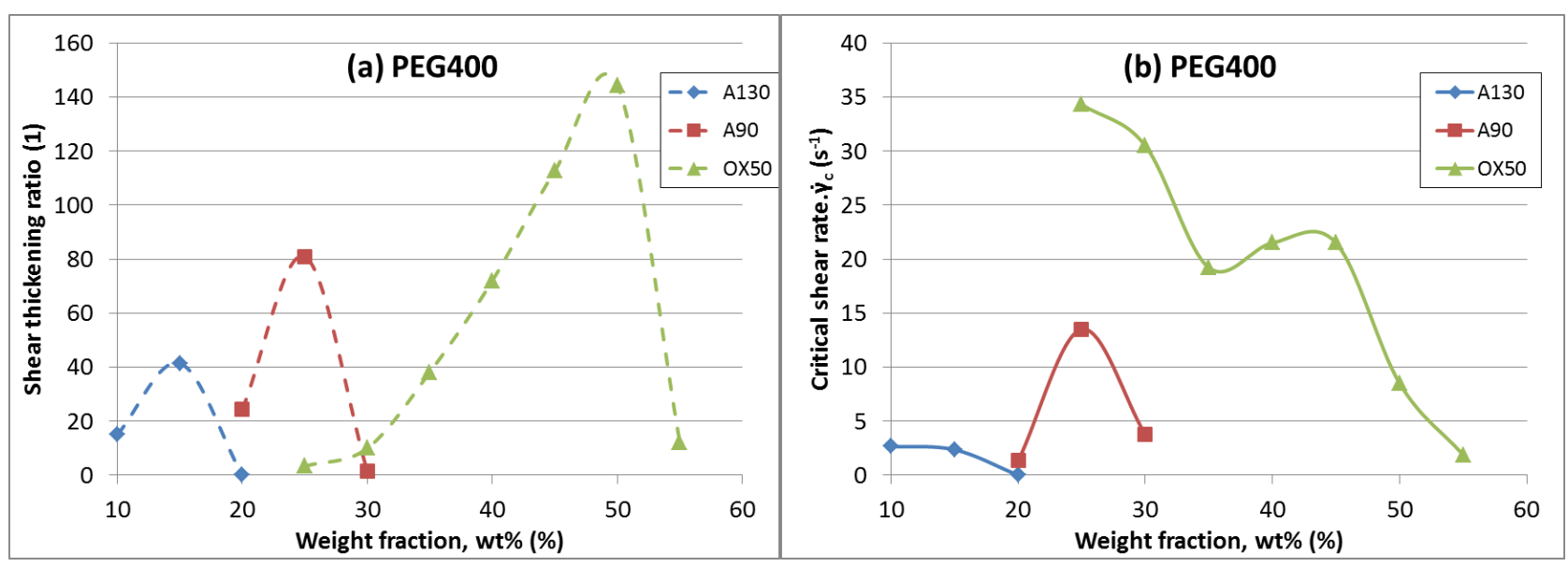

Figure 8. Shear thickening ratio (a) and critical shear rate (b) measured as a function of weight fraction mixed in PEG400 carrier fluid under steady shear conditions

By increasing the particle size, we are able to increase the weight fraction before attaining this 'Effective surface area', allowing us to maximise weight fraction within the STF, reduce interparticle distancing whilst allowing for sufficient space for particle rearrangement under shear stress. This would result in a higher shear thickening effect and lower $\dot{\gamma}_{c}$ due to the reduced external forces required to overcome particle repulsion forces. Another interesting relation is the effect of increasing particle size resulted in overall increased shear thickening performance and increased critical shear rate values.

In terms of potential applications, using larger particles will amplify the shear thickening effect and critical shear rate at the compromise of using more particles. By analyzing the requirements of the STF in mechanical applications, a suitable STF mixture can be fabricated that satisfy the application's thickening requirements and the shear force range at which it occurs. 


\section{Carrier fluid chemistry}

Observing the effect of varying liquid chemistry with equivalent molecular weight (PEG400 vs. PPG400) was explored experimentally. With PPG400, the shear thickening effect was found to occur at lower shear rates and at significantly larger magnitudes than PEG400. It was also observed that there was very little change in $\dot{\gamma}_{c}$ with changing weight fraction in PPG400 (Figure 7).

A possible theory as to why STFs with PPG attain shear thickening sooner is the relatively smaller DP which could affect the thickness of the solvation layer formed around the particles in the STF. This can attribute to smaller interparticle distancing and weaker particle-particle repulsion which results in less external force required for initiating shear thickening. Another possible suggestion is the composition of PEG compared to PPG. The only difference between these two polymers is an additional methyl branch within the PPG monomer, resulting in more rigid behavior under flow. More rigid polymer chains can typically lead to increased spacing between adjacent chains and reduced entanglement allowing for easier rearrangement whilst under shear flow. Therefore, ease of polymer flow will result in easier particle rearrangement to attain the shear thickening effect at lower shear rates. Comparing both STF mixtures with differing carrier fluids as shown in Figure 6, a similar trend is observed in which the rheological response of STFs containing PPG400 shows lower $\dot{\gamma}_{c}$ but higher STR and vice versa for STFs containing PEG400. Another interesting observation is the equal optimum weight fractions observed in both PEG400 and PPG400. The difference in rigidity of the carrier fluid chemical composition has little effect on the 'effective surface area' at which the same amount of particles added to both PEG400 and PPG400 will exhibit the maximum shear thickening effect and $\dot{\gamma}_{c}$ of both STFs. 
Comparing carrier fluids with similar chemical composition but with varying molecular weights (PPG400 vs. PPG725) in Figure 7 showed that STFs with higher molecular weight carrier fluid resulted in significantly lower STR and lower optimum weight fraction. These findings are consistent with those reported by $\mathrm{Xu}$ et al. [28]. EG and PEG are commonly used in STF research and their mixtures with silica particles usually exhibit shear thickening when mixed with $[2,4-6,28,30-32]$. The main difference differentiating PEG and EG is that PEG is a polymer of EG with a much higher degree of polymerization (DP) which could affect fluid viscosity. The molecular weight of a polymer is proportional to the DP, which could result in heavier polymer chains to form larger solvation layers on the particles, increasing interparticle distancing and repulsion forces. Another possible explanation is the increased DP resulted in entanglement, which hindered the formation of Hydroclusters under shear stress. However, by observing Figure $7\left(\right.$ b) the $\dot{\gamma}_{c}$ of both PPG400 and PPG725 show minute changes. One could infer the effect of decreasing the molecular weight of the carrier fluid would increase the shear thickening effect while the shear rate at which thickening commences remains relatively stable.

\section{Validity with the Modified Cox-Merz Rule}

Several previous investigations attempted to relate the shear thickening effect of STFs under steady shear conditions to those under dynamic (oscillatory) shear conditions using the Modified Cox-Merz theory (MCM) [24, 33-35], mathematically represented as:

$$
\eta(\dot{\gamma})=\eta^{*}\left(\omega \gamma_{0}\right)
$$


This relationship allows us to characterise a similar thickening effect under oscillatory shear flow, refereed as shear stiffening effect. Both $\mathrm{STF}_{\mathrm{PEG}}$ and $\mathrm{STF}_{\mathrm{PPG}}$ were tested under dynamic testing conditions. In which the amplitude shear strain, $\gamma_{0}$, is provided whilst the frequency is gradually increased. The results are shown in Figure 9.

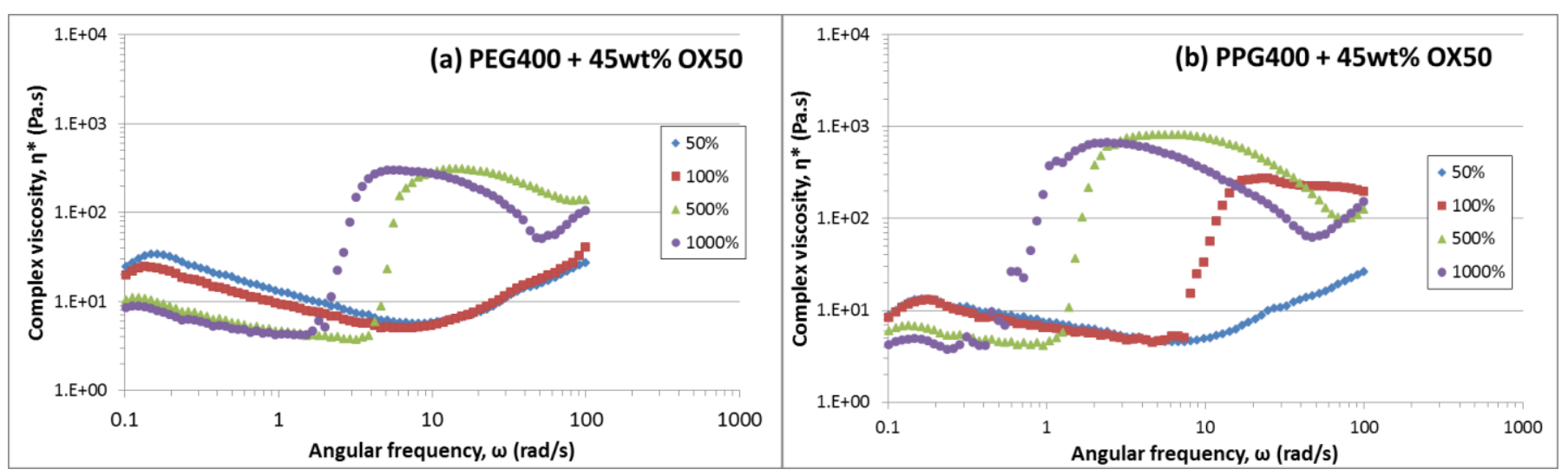

Figure 9. Dynamic frequency tests conducted on $\operatorname{STF}_{\mathrm{PEG}}$ (a) and $\operatorname{STF}_{\mathrm{PPG}}$ (b). Each data series represents the strain amplitude $\left(\gamma_{0}\right)$ applied.

Under comparison, both $\mathrm{STF}_{\mathrm{PEG}}$ and $\mathrm{STF}_{\mathrm{PPG}}$ behave differently as they do under steady shear conditions. $\mathrm{STF}_{\mathrm{PEG}}$ begins to show signs of shear stiffening when $\gamma_{0} \geq 500 \%$ whereas in $\mathrm{STF}_{\mathrm{PPG}}$ shear stiffening is apparent at $\gamma_{0} \geq 100 \%$ which also shows a relative increase in the ratio of $\eta_{\max }^{*} / \eta_{c}^{*}$. As observed previously, $\mathrm{STF}_{\mathrm{PPG}}$ was observed to exhibit shear thickening at lower shear rates and greater magnitudes than $\mathrm{STF}_{\mathrm{PEG}}$, which also appears evidently under oscillatory condition. Further analysis was completed by calculating the dynamic shear rate $\left(\omega \gamma_{0}\right)$ and comparing with steady shear results to observe the validity of the MCM rule (Figure 10). 

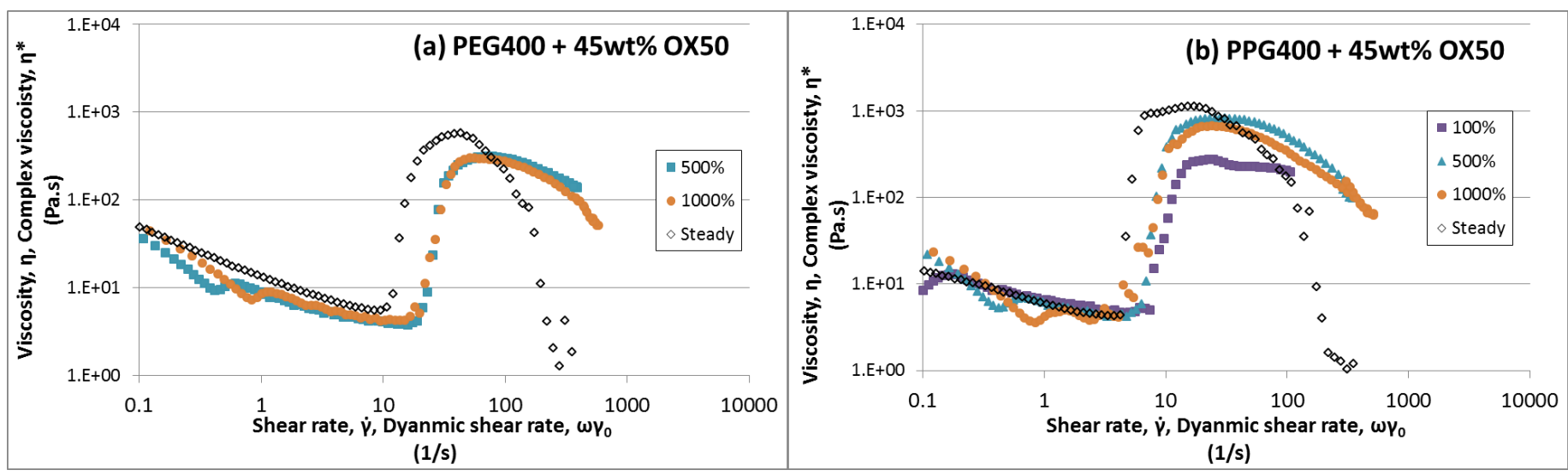

Figure 10. Correlation of steady and dynamic shear test data of $\operatorname{STF}_{\mathrm{PEG}}$ (a) and $\operatorname{STF}_{\mathrm{PPG}}$ (b) using MCM rule. Each data series represents the strain amplitude $\left(\gamma_{0}\right)$ applied.

The MCM model applies to a limited extent in both STFs but only at higher strain amplitudes $\left(\gamma_{0} \geq 500 \%\right)$. The dynamic shear data fits relatively well with the shear data as the deviation of $\dot{\gamma}_{c}$ with $\omega \gamma_{0}$ is relatively minor. The significance of these findings can allow for future research to extrapolate findings from steady shear tests to estimate the STF's shear stiffening performance under dynamic testing.

\section{Conclusions}

The effect of varying particle and liquid parameters on the resultant shear thickening effect was quantified. For each STF, there existed an optimum weight fraction that depended on the size of the fumed silica and larger particles resulted in the increased shear thickening effect and critical shear rate. The chemical properties of carrier fluids had a significant effect on the shear thickening properties by adjusting the critical shear rate and the shear thickening ratio. It was proposed that the mentioned variables affect the formation of solvation layer around the particles and interparticle distancing via polymer lubrication. Two STFs were selected for further testing based on their improved rheological behaviour to observe whether they conform to the Modified Cox-Merz rule and relate steady shear and dynamic shear conditions. Both STF's conformed to the rule 
which showed a relationship between shear-thickening and shear-stiffening at high strain amplitudes $\left(\gamma_{0} \geq 500 \%\right)$. These theories and suggestions into the effect of varying material properties on rheology aim at providing the ability to 'tailor' STFs for potential applications and future testing will be planned to observe the effect of differing critical shear rate and shear thickening ratios on current and potential real world situations.

\section{Acknowledgments}

The authors acknowledge AEROSIL® for supplying fumed silica samples for testing and Sigma-Aldrich ${ }^{\circledR}$ for supplying the various carrier fluids used for testing. 


\section{References}

1. Gong, X., et al., Study of the knife stab and puncture-resistant performance for shear thickening fluid enhanced fabric. Journal of Composite Materials, 2014. 48(6): p. 641-657.

2. Hassan, T.A., V.K. Rangari, and S. Jeelani, Synthesis, processing and characterization of shear thickening fluid (STF) impregnated fabric composites. Materials Science and Engineering A, 2010. 527(12): p. 2892-2899.

3. Lee, B.W. and C.G. Kim, Computational analysis of shear thickening fluid impregnated fabrics subjected to ballistic impacts. Advanced Composite Materials, 2012. 21(2): p. 177192.

4. Lee, Y.S., E.D. Wetzel, and N.J. Wagner, The ballistic impact characteristics of Kevlar ${ }^{\circledast}$ woven fabrics impregnated with a colloidal shear thickening fluid. Journal of Materials Science, 2003. 38(13): p. 2825-2833.

5. Li, X., et al., Preparation of body armour material of Kevlar fabric treated with colloidal silica nanocomposite. Plastics, Rubber and Composites, 2008. 37(5-6): p. 223-226.

6. Petel, O.E., et al., The effect of particle strength on the ballistic resistance of shear thickening fluids. Applied Physics Letters, 2013. 102(6).

7. Srivastava, A., A. Majumdar, and B.S. Butola, Improving the Impact Resistance of Textile Structures by using Shear Thickening Fluids: A Review. Critical Reviews in Solid State and Materials Sciences, 2012. 37(2): p. 115-129.

8. Wetzel, E.D., et al. The effect of rheological parameters on the ballistic properties of shear thickening fluid (STF)-Kevlar composites. in AIP Conference Proceedings. 2004. IOP INSTITUTE OF PHYSICS PUBLISHING LTD.

9. Zhao, J., et al. The stab resistant properties of Kevlar/STF composites. in Proceedings of SPIE - The International Society for Optical Engineering. 2012.

10. Zhao, J., et al., Effect of SiO 2 particle size on stab resistant properties of STF/Kevlar composites. Fuhe Cailiao Xuebao/Acta Materiae Compositae Sinica, 2012. 29(1): p. 54-61.

11. The ballistic impact characteristics of Kevlar ${ }^{\circledR}$ woven fabrics impregnated with a colloidal shear thickening fluid. J. Mater. Sci., 2003. 38(13): p. 2825.

12. Neagu, R.C., P.E. Bourban, and J.A.E. Månson, Micromechanics and damping properties of composites integrating shear thickening fluids. Composites Science and Technology, 2009. 69(3-4): p. 515-522.

13. Fischer, C., et al., Dynamic properties of sandwich structures with integrated shearthickening fluids. Smart Materials and Structures, 2006. 15(5): p. 1467.

14. Peng, G.R., et al., Experimental and modeling study of viscoelastic behaviors of magnetorheological shear thickening fluids. Korea Australia Rheology Journal, 2014. 26(2): p. 149158.

15. Jiang, J., et al., Shear thinning and shear thickening characteristics in electrorheological fluids. Smart Materials and Structures, 2014. 23(1).

16. Zhang, X., W. Li, and X. Gong, Thixotropy of MR shear-thickening fluids. Smart Materials and Structures, 2010. 19(12).

17. Hoffman, R.L., DISCONTINUOUS AND DILATANT VISCOSITY BEHAVIOR IN CONCENTRATED SUSPENSIONS--1. OBSERVATION OF A FLOW INSTABILITY. Trans Soc Rheol, 1972. 16(1): p. 155-173.

18. Hoffman, R.L., Discontinuous and dilatant viscosity behavior in concentrated suspensions. II. Theory and experimental tests. Journal of Colloid And Interface Science, 1974. 46(3): p. 491-506.

19. Boersma, W.H., J. Laven, and H.N. Stein, Computer simulations of shear thickening of concentrated dispersions. Journal of Rheology, 1995. 39(5): p. 841-860.

20. Durlofsky, L., J.F. Brady, and G. Bossis, DYNAMIC SIMULATION OF HYDRODYNAMICALLY INTERACTING PARTICLES. Journal of Fluid Mechanics, 1987. 180: p. 21-49. 
21. Bergenholtz, J., J.F. Brady, and M. Vicic, The non-Newtonian rheology of dilute colloidal suspensions. Journal of Fluid Mechanics, 2002. 456: p. 239-275.

22. Lee, J.D., J.H. So, and S.M. Yang, Rheological behavior and stability of concentrated silica suspensions. Journal of Rheology, 1999. 43(5): p. 1117-1140.

23. Barnes, H.A., Shear-thickening ('dilatancy') in suspensions of nonaggregating solid particles dispersed in Newtonian liquids. Journal of Rheology, 1989. 33(2): p. 329-366.

24. Raghavan, S.R. and S.A. Khan, Shear-thickening response of fumed silica suspensions under steady and oscillatory shear. Journal of Colloid and Interface Science, 1997. 185(1): p. 5767.

25. Wang, R. and S.L. Wunder, Effects of Silanol Density, Distribution, and Hydration State of Fumed Silica on the Formation of Self-Assembled Monolayers of $n$-Octadecyltrichlorosilane. Langmuir, 2000. 16(11): p. 5008-5016.

26. Gun'ko, V.M., et al., Morphology and surface properties of fumed silicas. Journal of Colloid and Interface Science, 2005. 289(2): p. 427-445.

27. Raghavan, S.R., H.J. Walls, and S.A. Khan, Rheology of silica dispersions in organic liquids: New evidence for solvation forces dictated by hydrogen bonding. Langmuir, 2000. 16(21): p. 7920-7930.

28. Liu, X.Q., et al., Temperature induced gelation transition of a fumed silica/PEG shear thickening fluid. RSC Advances, 2015. 5(24): p. 18367-18374.

29. He, Q., et al., Shear thickening of suspensions of porous silica nanoparticles. Journal of Materials Science, 2015. 50(18): p. 6041-6049.

30. $\mathrm{Xu}, \mathrm{Y}$.L., et al., Shear thickening fluids based on additives with different concentrations and molecular chain lengths. Chinese Journal of Chemical Physics, 2010. 23(3): p. 342-346.

31. Shan, L., et al., Influences of medium and temperature on the shear thickening behavior of nano fumed silica colloids. Wuli Xuebao/Acta Physica Sinica, 2015. 64(6).

32. Baird, J.A., et al., Effect of molecular weight, temperature, and additives on the moisture sorption properties of polyethylene glycol. Journal of Pharmaceutical Sciences, 2010. 99(1): p. 154-168.

33. Chellamuthu, M., E.M. Arndt, and J.P. Rothstein, Extensional rheology of shear-thickening nanoparticle suspensions. Soft Matter, 2009. 5(10): p. 2117-2124.

34. Fischer, C., et al., Pre- and post-transition behavior of shear-thickening fluids in oscillating shear. Rheologica Acta, 2007. 46(8): p. 1099-1108.

35. Galindo-Rosales, F.J., F.J. Rubio-Hernández, and J.F. Velázquez-Navarro, Shear-thickening behavior of Aerosil ${ }^{\circledR}$ R816 nanoparticles suspensions in polar organic liquids. Rheologica Acta, 2009. 48(6): p. 699-708. 